\title{
Resources Management of Mobile Network IEEE 802.16e WiMAX
}

\author{
Mubarak Elamin Elmubarak Daleel 1 \\ ${ }^{1}$ Department of Information Systems \\ Faculty of Computing \& Information Technology, \\ University of Jeddah, Kingdom of Saudi Arabia \\ ${ }^{2}$ Department of Computer Science, Faculty of Computer \\ Science \& Information Technology, Alzaeim Alazhari \\ University, Sudan
}

\author{
Marwa Eltigani Abubakar Ali ${ }^{3}$ \\ ${ }^{3}$ Faculty of Engineering \\ Al Neelain University, \\ Sudan
}

\begin{abstract}
The evolution of the world of telecommunications towards the mobile multimedia following the technological advances has demonstrated that to provide access to the network is no longer sufficient. The need for users is to access value-added multimedia services in their own home environment regardless of how they access the systems. Multimedia services require high transfer rates and have quality service requirements. They must coexist with services with real time constraints such as the voice service which does not tolerate variation of the delay between sending and receiving packets. The guarantee of these services by the operator becomes much more difficult in the technologies that take into account the mobility of the users.

This paper studies the IEEE802.16e system according to the continuous modeling case. A model of the IEEE802.16e cell is proposed and allows the decomposition of the cell according to the principle of the AMC adaptive modulation and coding technique. The model is based on an admission control mechanism in the presence of two types of real-time and nonreal-time traffic. The model is based on a new CAC strategy with intra-cell mobility and gives the same QoS for the calls of this traffic by favoring the calls in progress on the new arrivals.
\end{abstract}

Keywords-Wireless Networks; IEEE 802.16; WiMAX; Radio Resource Allocation; Mobility; Admission Control

\section{INTRODUCTION}

Mobile communications systems have evolved considerably in recent years. This has encouraged the deployment of several multi-technology cellular systems or networks. Quality of service (QoS) for mobile users improves across systems. Third generation (3G) systems, such as UMTS, offer better quality of service compared to second generation (2G) systems such as GSM. For example, 3.5G (HSDPA) systems improve the $3 \mathrm{G}$ network throughput on the downlink to meet the requirements of the new services. In addition, fourth-generation (4G) networks, such as WiMAX (IEEE802.16e) [1], extend cell coverage while delivering superior throughput. This new development further improves the accessibility of Internet services. Migration of services from the Internet to mobile networks is a major challenge for telecommunications research.

In particular, efforts have been made to optimize the quality of service and to differentiate between real-time and best-effort or non-real-time services. It is a service and content subject to constraints of availability of radio resources and network load, while maintaining the semantics and offering a rendering in accordance with users' expectations.

New mechanisms are needed to differentiate services and provide the quality of service required. These mechanisms include admission controls, resource reservation protocols, and packet scheduling policies. The challenge for nextgeneration networks is to maintain high bit rates and quality of service on radio links (up and down) that are unreliable for transmissions. CDMA is the most advanced technique of multiplexing, intended to be used especially on third generation mobile telephony networks such as UMTS [2]. Whereas the previous techniques of frequency multiplexing (FDMA) and time division multiplexing (TDMA) essentially consisted of dividing a physical quantity (a frequency bandwidth or the time that elapses) into individual "slots", the CDMA does not fix No prior static distribution of these resources and resembles computerized packet transmission techniques.

This technique was chosen because of the constraints specific to the $3 \mathrm{G}$ radio interface: variable rate, variable quality of service (multiservice traffic environment). It allows simultaneous access of multiple users at very high speeds and services (sound, image, multimedia, ...). There are nevertheless different CDMA variants. The one on which the UMTS standard called WCDMA is based. The speed promised by UMTS in theory is $2 \mathrm{Mbits} / \mathrm{s}$, but it is never reached in practice because of the $3 \mathrm{G}$ network limitations. A WCDMA software extension called HSDPA was introduced to improve the downlink rate where more information will be transported. With the HSDPA [3] technique the throughput can reach $14 \mathrm{Mbps}$ in the $3.5 \mathrm{G}$ network or HSP +. To better manage radio resources by the operator with more flexibility in terms of quality of service and throughput, a new OFDMA multiple access method has become the basis for nextgeneration networks such as IEE80.16e and LTE. The WiMAX network allows a theoretical throughput of 70 Mbits/s over a maximum radius of $50 \mathrm{~km}$ while relying on new techniques such as the adaptive modulation and coding scheme depending on the state of the radio channel associated with a mobile. LTE promises a very high bandwidth of up to $100 \mathrm{Mbps}$ as well as wide accessibility of coverage $(100 \mathrm{~km}$ in rural areas). 
In this paper, we introduce a new admission control mechanism into the IEEE802.16e (WiMAX) network with two types of real-time and non-real-time traffic. The continuous case of the multiservice IEEE 802.16e system is studied. We develop a new resource allocation mechanism that takes into account intra-cell mobility of users. We consider a mobility model called RWP (Random Waypoint).

The remainder of this paper is divided into six sections. After introducing, the evolution of communication standards is presented in Section 2. Section 3 describes the standard IEEE802.16 (WiMAX) and its service classes and QoS. Section 4, describes related work on the capacity and admission control models of an HSDPA and IEEE802.16 cell. In section 5, an analysis of the IEEE 802.16e system is presented. Finally, this paper is concluded in Section 6.

\section{MOBILE COMMUNICATION SYSTEMS}

The eighties witnessed the birth of first generation, fully analog cellular mobile phone systems, such as Nordic Mobile Telephony (NMT), Advanced Mobile Phone Service (AMPS), Total Access Cellular System (TACS) or the Radiocom 2000.

Nevertheless, reduced spectral resources, short battery life and terminal cost were obstacles that limited the early development of first generation mobile phones. The second generation of cellular systems emerged in the early 1990s with DECT (Digital Enhanced Cordless Telecommunications), GSM (Global System for Mobile Communications, Europe), PDC (Japan) and PCS (United States). Initially designed for speech and data transmission applications at low bit rates (9600 bits/s) around frequencies (935-960 MHz) or (890-915 $\mathrm{MHz}$ ), this standard has allowed for the growth of mobile communications. The performance (spectral efficiency, safety, functionality) of the second generation of cellular systems is greater than that of the first generation. An important contribution to these systems has been the modification of the method of data access, and the birth of the intermediate generation named $2.5 \mathrm{G}$, embodied by the General Packet Radio Service (GPRS) system. Another form of evolution of second-generation cell systems is embodied in EDGE (Enhanced Data Rate for GSM Evolution) systems; they use a new modulation, optimizing the effective rate at the GSM interface, allowing to reach speeds of $384 \mathrm{Kbps} / \mathrm{s}$ at the maximum. Different continents have developed systems that are fairly similar in principle, but sometimes different in their realization. It is therefore within the framework of a global homogenization of standards that the idea of a third generation of cellular systems was born. The third generation (3G) of cellular systems, often assimilated in Europe to UMTS (Universal Mobile Telecommunication Services) [2], has been mainly designed and imagined to satisfy multimedia data routing (Internet access, corporate networks , Courier services, videoconferencing, games, etc.). In the same way as the GPRS and EDGE allowed, without modifying the basic principle of communications, their generation of cellular systems to evolve in terms of efficiency. The HSDPA (High-Speed Downlink Packet Access) [3] is already considered the standard 3.5G mobile telecommunications. While the maximum bit rate allowed on a UMTS link is $2 \mathrm{Mbps}$ for a $5 \mathrm{MHz}$ bandwidth, the HSDPA, thanks to its 16-QAM modulation, allows for 10
Mbits/s. Thus, thanks to the introduction of the new 64-QAM modulation in Release 7, the theoretical rate of $14 \mathrm{Mbits} / \mathrm{s}$ is possible via the HSDPA network.

In the same sense, IEEE802.16 (WiMAX) 4G network allows a theoretical rate of 70 Mbits / s over a maximum radius of $50 \mathrm{~km}$ while being based on different modulations depending on the state of the radio channel and New ways of accessing the channel. The performance of the system in terms of throughput is much greater than that provided by the previous systems, thanks to these modulations (QPSK, 16QAM, 64-QAM). The WiMAX Mobile standard allows services such as VoIP communication (Telephony over IP network) on mobile phones or access to mobile services at high speeds. Its equivalent in Europe is the Hiper-MAN. In addition, the main purpose of new communication technologies is to eliminate cables and allow transmission (voice, data, etc.) via a radio link. Fig. 1 summarizes the evolution of wireless networks.

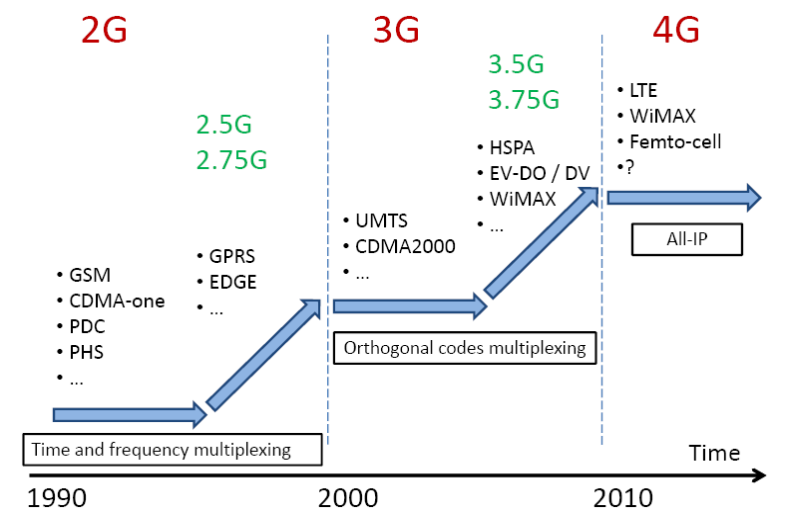

Fig. 1. Evolution of wireless networks

Thus, the rapid development of wireless communications and the emergence of new standards call for convergence towards the fourth generation of mobile communications. Originally planned for the years 2010, it would seem to present its very first commercial achievements slightly earlier. This advance in relation to the forecasts is due in particular to the fact that the fourth generation will not be the outcome of a revolution in communications (as in the second and third generation), but rather the convergence of different Standards, applications and products. Indeed, it seems relevant to consider $4 \mathrm{G}$ as the convergence of standards and technologies covered by $3 \mathrm{G}$ and wireless local area networks (WLANs). The goal of $4 \mathrm{G}$ is to improve the performance of the third generation, without fundamentally changing the content or applications originally planned for $3 \mathrm{G}$.

\section{IEEE 802.16E (WIMAX)}

\section{A. Presentation of IEEE 802.16e (WiMAX)}

The Wimax [1] is a technology mainly used for MAN (Metropolitan Area Network). It allows, in particular, rural areas to have a broadband internet connection. The objective of WiMAX is to provide a broadband internet connection over a coverage area several kilometers radius. The theoretical speeds of WiMAX are $70 \mathrm{Mbits} / \mathrm{s}$ with a range of 50 kilometers. In practice in the worst case scenario, ie in NLOS 
"Non Line Of Sight", the rate reaches 12 Mbits/s up to 4.5 kilometers. The WiMAX works in point-to-multipoint mode, that is to say the infrastructure mode known for WiFi or the same functioning as the $2 \mathrm{G}, 3 \mathrm{G}$ technologies of mobile telephony. Fig. 2 presents the IEEE 802.16 network architecture. As in 2G, a base station called BTS (Base Transceiver Station) or BS (Base Station) transmits to the clients and receives their requests and transmits them to the network of the access provider. An overview of a backbone mesh network and connections to WiFi, WiMAX, and wireless cellular networks is presented in Fig. 3. Several variants of standard have been proposed, modified and ratified as summarized in Table 1.

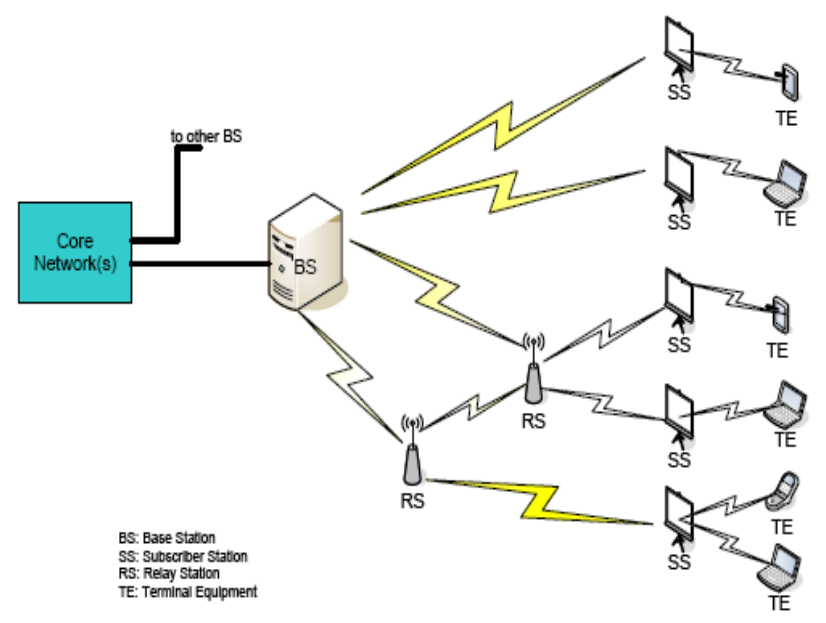

Fig. 2. IEEE 802.16 network architecture

We are interested in the IEEE802.16e standard [4] which uses in its physical layer the OFDMA method initially developed by Sari and Karam [5] as a multiple access method. In order to report specific events, the mobiles transmit Ranging signals to the base station. These messages are of different types depending on the type of event to be reported. The signaling transmission uses a set of 256 orthogonal codes (CDMA codes) encoded on 144 bits. These codes are distributed among the four possible types of signaling. A mobile unit wishing to transmit one of these signals randomly selects one of the codes available in the subset specific to its signaling family. This code is then transmitted via binary phase modulation (BPSK). The IEEE802.16e standard establishes four signaling categories: Initial Ranging (IR) initialization, Hand-Over Ranging (HR) migration request, Bandwidth Request Ranging (BR) request, and request Periodic Ranging (PR).

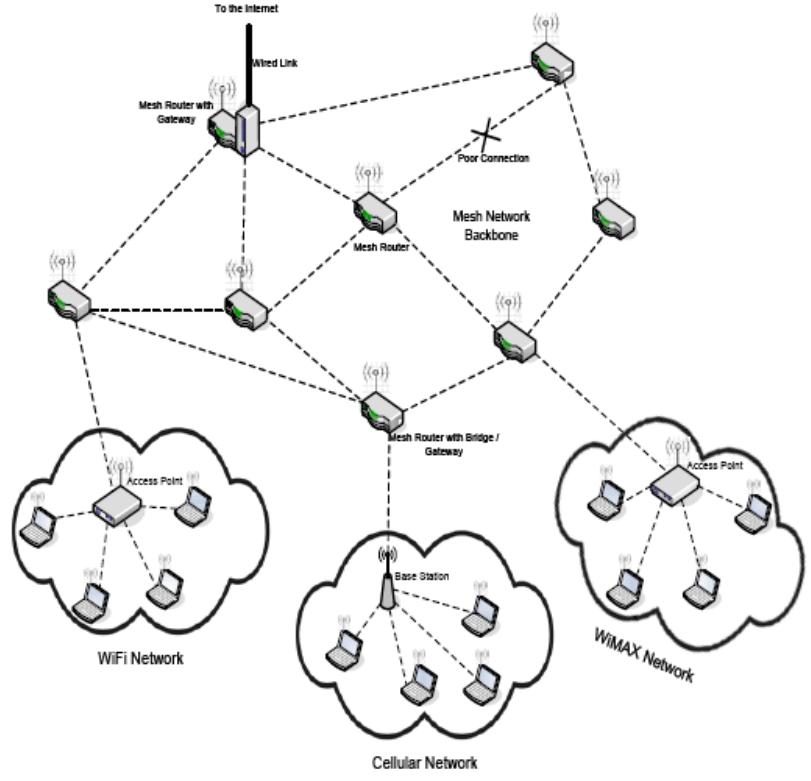

Fig. 3. Connections to Backbones mesh networks

TABLE. I. IEEE802.16 STANDARD HISTORY

\begin{tabular}{|l|l|}
\hline Standard IEEE802.16 & Frequency Band (FB) \\
\hline IEEE std 802.16 & $\begin{array}{l}\text { Metropolitan wireless networks FB } \\
\text { greater than 10 GHz. }\end{array}$ \\
\hline IEEE std 802.16a & $\begin{array}{l}\text { Metropolitan wireless networks FB } \\
\text { between 2 and 11 GHz. }\end{array}$ \\
\hline IEEE 802.16b & $\begin{array}{l}\text { Metropolitan wireless networks FB } \\
\text { between 10 and 60 GHz. }\end{array}$ \\
\hline IEEE std 802.16c & $\begin{array}{l}\text { Metropolitan wireless networks FB in } \\
\text { free bands. }\end{array}$ \\
\hline $\begin{array}{l}\text { IEEE 802.16d (IEEE std } \\
\text { 802.16-2004) }\end{array}$ & $\begin{array}{l}\text { Revision integrating standards 802.16, } \\
802.16 a \text { and 802.16c. }\end{array}$ \\
\hline IEEE std 802.16e & $\begin{array}{l}\text { Metropolitan wireless networks with } \\
\text { mobile customers. }\end{array}$ \\
\hline IEEE std 802.16f & Networks (mesh network). \\
\hline
\end{tabular}

B. Service classes and QoS defined by the IEEE802.16 standard

Scheduling services consist of the mechanisms supported by the Medium Access Control layer (MAC) for data transport. Each connection is associated with a single class of service. These service classes involve a particular set of parameters that define the QoS associated with the connection. Four classes of services are proposed in the standard $[4,6]$ : the unsolicited acceptance service (UGS), the real-time service (rtPS), the data transfer service (nrtPS) and interactive traffic (BE). In addition, a last class has recently integrated the standard constituting an extension of the real-time service (ertPS). They are defined as follows: 
- Unsolicited Grant Services (UGS): The UGS class is intended to support real-time data streams characterized by a fixed size of packets received periodically. This class is therefore intended for T1 / E1 services and voice over IP (VoIP) without silence suppression. The associated QoS parameters are then the maximum and minimum traffic to be reserved for traffic (often the same), maximum delay, tolerated jitter, update policy. The mobile maintains an optimal delay by the fact that no request is sent to the contention sub-channel once the service is engaged. This class of traffic therefore eliminates the data and delays caused by repeated bandwidth requests. The BS then periodically reserves to the mobile part of the available radio resources. The reserved quantity is initially equal to the maximum requested flow. This class is dedicated to real-time streams. The frames are of fixed size and emit regularly;

- Real-time service rtPS (real-time Polling Services): The rtPS class is designed to handle real-time traffic for which the size of data stream packets is variable and at regular intervals. Also, this class is used for video traffic such as MPEG, H.263. The Quality of Service settings are based on a minimum bandwidth for video traffic, a maximum acceptable bit rate, a maximum delay, and an update periodicity. The rptenabled mobile has the ability to update its performance parameters by periodically incorporating new queue resource requests into the data packets. During the restraint interval. This class therefore respects time-sensitive traffics by avoiding the collisions involved in the contention interval to update requests. In addition, the mobile has the opportunity to indicate to the BS what periodicity of updating requires the traffic engaged. It allows real-time flows. The frames are of variable size and the emission may be irregular. This class is suitable, for example, for a video stream;

- Extension of the real time service ertPS (extended real time Polling Service): The ertPS class is intended to support real-time data streams characterized by a variable size of packets received periodically. This class is therefore intended for voice over IP (VoIP) services with silence suppression. The Quality of Service settings are based on a minimum bitrate for video traffic, a maximum acceptable bit rate, a maximum delay but no update timing indicator. The SS ertPS traffic has the ability to update its performance parameters by incorporating new resource requests at the queue of data packets or during the contention interval when necessary. This class therefore respects time-sensitive traffic by avoiding the collisions implied by the contention interval to update requests. Additionally, this class of traffic reduces signaling traffic and the delay time for dynamically allocated resources. In addition, variable resource allocation for ertPS calls avoids resource waste on uplink, as may be the case on UGS calls;
- NrtPS data transfer service (non-real time Polling Service): the nrtPS class must be able to manage traffic that is insensitive to the delay but requires a minimum throughput. The size of the packets can therefore be variable, as well as the delay between two packet transmissions. This class is therefore very suitable for the file transfer protocol (FTP). The Quality of Service settings are: minimum traffic flow to be reserved, maximum possible throughput for connection and traffic priority. Once the connection is initiated, the SS must imperatively issue a new bandwidth request in order to be able to claim another rate;

- BE (Best Effort) Interactive Service: The BE class is dedicated to traffic that does not require a particular level of performance. The QoS parameters are only related to the maximum possible throughput for connection, traffic priority, and query renewal policy. As for the previous class, the class engages a new connection for any new resource need. In this class nothing is guaranteed we send the data without guarantee of reception. Suitable for Internet browsing.

\section{RELATED WORKS}

This section describes the literature work associated with the models of capacity and admission control address the main means and tools for modeling the capacity of an HSDPA and IEEE802.16 cell with or without the management of resources in relation to mobility of users.

Numerous works have been developed in the literature to study the capacity of wireless networks. In [7], $\mathrm{Li}$ et al. Develops a downlink admission control of the broadband network WiMAX and HSDPA based on the adaptation of the radio link of the air interface. They decompose the cell into a finite number of regions taking into account a simple propagation model operating with only a path loss as a function of the distance between the mobile and the base station. They consider in their study a single class of service and model the system by the network of queues of type BCMP [8], [9] whose number of active users is fixed. The authors assume that the arrival rates of calls in each region only depend on the average number of mobile that move in that region. Thus, they consider that the average time of service depends only on the time that the mobile remains in the region.

The authors in [10] are interested in the resource management of HSDPA and WiMAX networks taking into account mobility. They present three RRM (Resource Management) resource management schemes in a cooperative system containing HSDPA and WiMAX and compare the performances in these schemes in terms of probability of blocking new calls, lost calls in progress and time files transfer. The authors rely on a Markov model with game theory for resource management in the presence of several regions of modulation efficiency.

Quality management and performance evaluation in the MAC layer of the IEEE 802.16e network were largely performed in simulation; And few analytical results have been proved in the literature. In [11], the authors analyze the 
performance of the random access protocol in an OFDMACDMA environment using contention intervals for connection engagement, as a function of the average MDT delay. In [12], the authors define a performance model based on an adaptive control of the size of each family of codes: initialization of connections (IR), periodic demand (PR) and bandwidth demand (BR), In order to improve the efficiency of competitive access.

Many previous studies assume that packets, or calls, arrive in the system following a Poisson process. This, in view of the simplification of the analytical model. Moreover, they consider that the incoming processes of any type of calls or packets are independent. Nevertheless, since in WiMAX, all mobiles share a finite number of CDMA codes, the arrivals of these calls should be dependent, as proved by [13]. Indeed, the authors develop a model of MAC access to signaling requests based on the different priority classes using the differentiation of the backoff parameters and the free codes for these classes. They consider the case of classification of connections based on partitioning codes between call classes with a sub-range of codes reserved for the real-time class and another under code range shared with that non-real time.

The work developed in [14], defines a QoS architecture associating a scheduler and an admission control algorithm. In a richer and more subtle way, the study [15] develops a successful and dynamic admission control algorithm in order to ensure the quality of service of each traffic class of the IEEE802.16e standard. The algorithm reserves the resources available for non-real-time traffic. These will then be rejected if the capacity of the cell is exceeded by the new request for resources. In parallel, other types of traffic will share the remaining resources. In addition, the allocation principle proposed here introduces a dynamic evolution called the degradation of resources. Indeed, the arrival of new real-time calls will result in the progressive and adaptive reduction of the remaining resources shared between non-real-time calls. Thus, the author gives the highest priority to UGS and rtPS traffic while maximizing the overall throughput by adapting the bandwidth to other traffic.

The work of [16] proposes an innovative sequencing of the allocation of resources between traffics. The principle of TRS (scheduling by timed omission) attributes the resources according to the modulation efficiency of each user. Based on the observation that users with the worst signal-to-noise ratio (SNR) generate more waste of radio resources, they will be allocated fewer communication resources and only at spaced intervals. This mechanism assumes that user mobility will enable them to take full advantage of the resources they need. This original resource allocation mechanism results in a significant gain in spectral efficiency. As a result, communications from nearby regions are quickly served, and in the context of a non-saturated network, this indirectly leads to better support for communications from more peripheral regions. This work has the particularity of taking into account the spectral efficiency of the users with respect to the radio resources allocated to them.

\section{ANALYSIS OF THE IEEE 802.16E SYSTEM}

In this section we are interested in the management of fourth generation network resources (WiMAX, known as 4G network) based on the IEEE 802.16e standard. One of the founding objectives of the WiMAX Forum is the search for interoperability: it is achieved through the channels of standardization and certification and is one of the major challenges of WiMAX. This is all the more important given that WiMAX is defined for a wide band of frequencies, from 2 to $66 \mathrm{GHz}$, in which there are existing technologies, such as Wi-Fi, which allows broadband, scopes and A wide variety of uses. This multiplicity of the frequency bands targeted, of the flows, reaches and possible uses, is besides the main pitfall that confronts the final consumer. The $4 \mathrm{G}$ network aims to achieve the convergence of existing networks such as Wi-Fi, UMTS, etc.

The services offered by WiMAX are classified according to the parameters defining the quality of service associated with a connection. Four classes of services are offered in the standard: Unsolicited Acceptance Service (UGS), Real Time Service (rtPS), Data Transfer Service (nrtPS) and Interactive Traffic (BE). Two main classes of service were considered during this work: real time (RT), corresponding to the classes UGS where rtPS, and non-real time or best effort (NRT), corresponding to the classes nrtPS and BE. Thus, we consider the case where the partitioning codes allocated to the connection requests are distributed between the call classes. We also consider in our study a single WiMAX/IEEE802.16 cell decomposed into $r$ concentric regions and each uses a different adaptive modulation (AMC) as recommended in [4].

The objective of this paper is to model the IEEE802.16e cell in a continuous case considering the behavior of mobile users. We propose an admission control strategy based on the Random Waypoint mobility model (RWP). This model allows us to realistically calculate call migration rates between regions of the cell, as indicated by [17]. In this admission control mechanism, the RT calls are characterized by the same bit rate. Thus, a call of type RT receives a number of subcarriers according to its modulation efficiency and the targeted region. The system accepts RT calls until the capacity is exceeded. Thus, they could be blocked by the system. On the other hand, calls of type $\mathrm{BE}$ receive the same number of subcarriers. Since the BE calls tolerate the rate reduction, they will use the carriers left by the current RT calls fairly according to Processor Sharing (PS) [18]. Also, the bit rate of a BE call depends on its region (ie modulation), but also on the number of $\mathrm{BE}$ calls in progress. With this resource management policy for BE calls, the system will never block a call of this type.

Finally, the duration of calls RT is independent of the resources consumed, and therefore the time that will remain an RT call in the system depends solely on the behavior of the user. Conversely, BE calls remain in the system according to the consumption of the resources, and therefore the more the number of sub-carriers increases, the faster the BE call ends its service. 
In addition, our CAC mechanisms seek to reduce the likelihood of loss: the likelihood that a call in progress will be rejected because of its mobility and that it changes the region. As explained above, the call consumes the bandwidth as a function of the modulation used. By migrating to a far region, a call may require additional bandwidth. This could result in a loss due to lack of available resources. To prevent this loss, our CAC mechanisms introduce some of the bandwidth reserved for mobile calls. This reservation is intended to meet the need for additional resources required for migration to areas where modulation is higher.

We consider, during our analysis and without loss of generality, adaptive modulation and coding (AMC) with only the pathloss. Depending on the latter, the OFDMA IEEE802.16e cell is decomposed into concentric $\mathrm{r}$ regions. Each region corresponds to a certain range of SNR values, and thus to an AMC, as shown in Fig. 4.

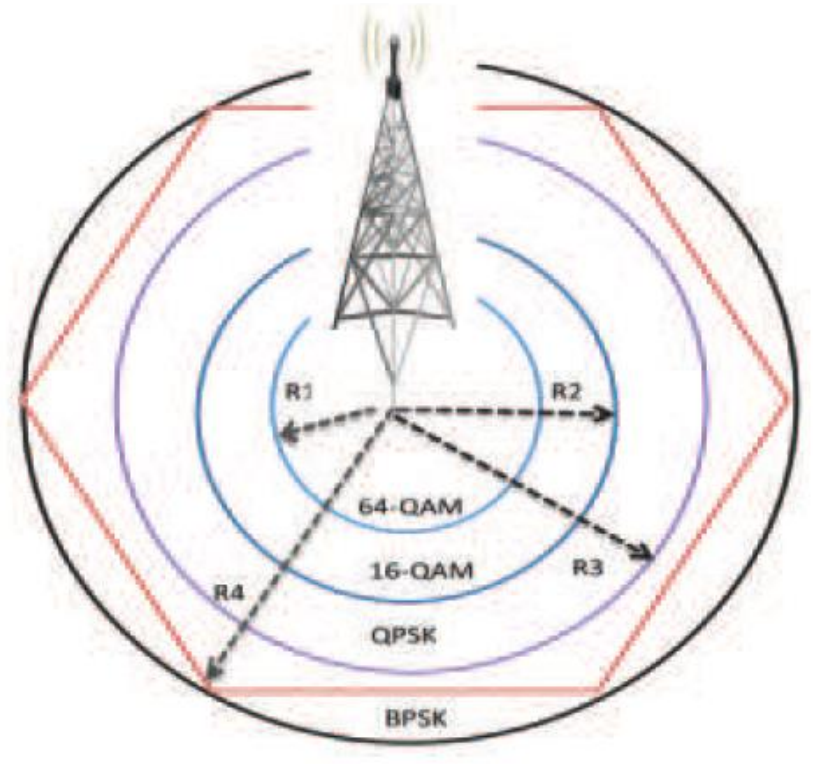

Fig. 4. The OFDMA IEEE802.16e sectored cell

We consider the following parameters:

- The OFDMA cell IEEE802.16e is decomposed into two concentric regions $(r=2)$ whose radii are $R l=$ $300 \mathrm{~m}$ and $R 2=600 \mathrm{~m}$.

- The modulations of these regions are 16-QAM 3/4 (e1 $=3 \mathrm{bits} / \mathrm{symbol})$ and QPSK $1 / 2$ (e2=1 bit / symbol) [19];

- The baud rate is $B=2666 \mathrm{symbol} / \mathrm{s}$;

- The block error rate $B L E R_{i}=0$;

- The number of frequencies is $K=48$;
- The call bit rate RT $R_{R T}$ is $128 \mathrm{Kbps}$ and that of calls $\mathrm{BE} R_{B E}$ is $384 \mathrm{Kbps}$ [20];

- The total capacity of system $L$ is 10 carriers;

- The average call duration RT is 120 s and the average download file size corresponds to $E(P a y)=5$ Mbits.

Finally, we assume that the mobiles or the users move according to the RWP process in the convex disk of radius $R_{z}$ $=900 \mathrm{~m}$. They randomly selected new speeds in each waypoint from a uniform velocity distribution in the interval $\left[v_{\min }, v_{\max }\right]$, where $v_{\min }=3 \mathrm{~km} / \mathrm{h}$ (low mobility) or $v_{\min }=20 \mathrm{~km} / \mathrm{h}$ (high mobility), $v_{\max }=90 \mathrm{~km} / \mathrm{h}$.

Fig. 5 shows the blocking probabilities for real-time traffic in both regions based on resource reservation for best effort calls. This figure clearly shows the impact of $L_{B E}$ and $L_{R T}^{m}$ on blocking probabilities in each region. Generally, blocking probabilities are greatly increased when the minimum reserve of resources for $\mathrm{BE}$ calls increases. In addition, this effect becomes more important by increasing the mobility management resource reservation. From this ratio, we could compute the ranges of values for $L_{B E}$ and $L_{R T}^{m}$ that satisfy a maximum probability blocking threshold.

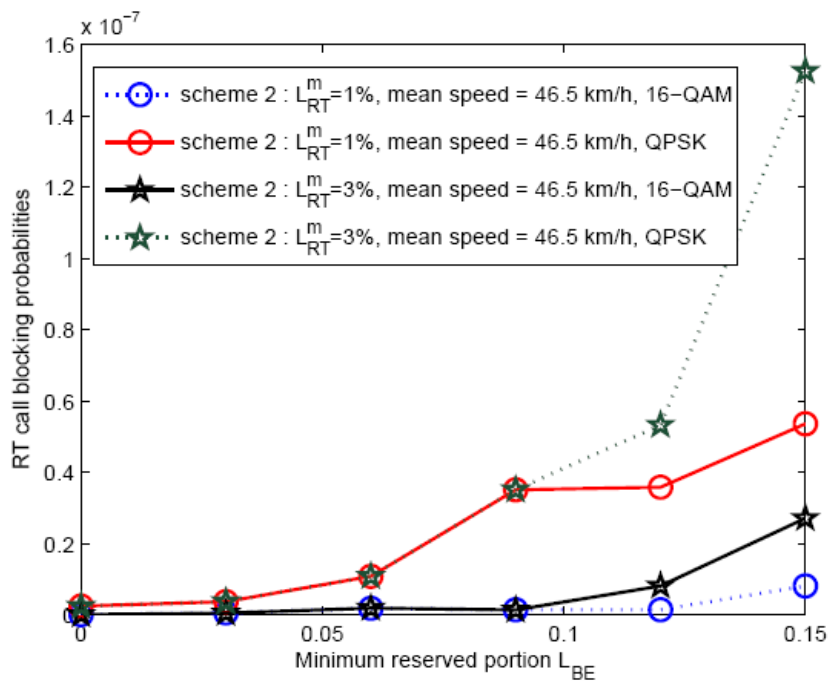

Fig. 5. Blocking probabilities for real-time traffic

Fig. 6 shows the loss probabilities for real-time traffic in the border region based on the minimum resource reservation for best effort calls. The figure shows how the reservation of resources $L_{R T}^{m}$ decreases the probability of loss via the minimum threshold reserved for calls BE. In this figure, the probability of loss is reduced when the $L_{B E}$ reservation is greater than ten percent. Thus we can determine the possible values for $L_{R T}^{m}$ arriving at a maximum probability of loss of calls RT maximum. 


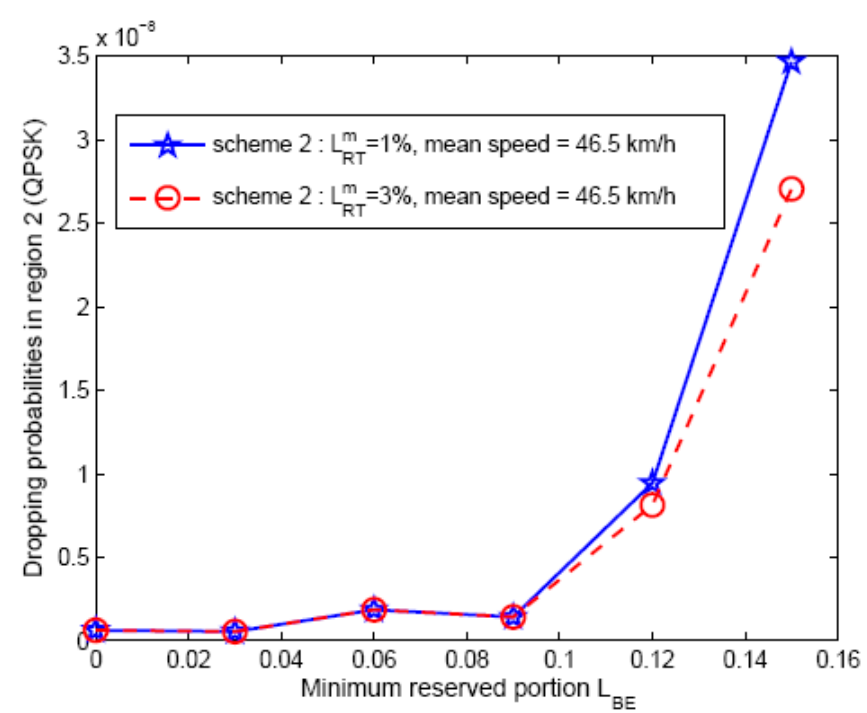

Fig. 6. RT calls loss probabilities

Finally, Fig. 7 shows the total flow rate of the cell. This figure show the impact of the different resource reservations for mobility on the increase of calls $\mathrm{BE}$ and the ease of the mobility of the calls. In Fig. 7 we can appreciate the important impact of the L_BE reservation. Indeed, by reserving $15 \%$ of the total capacity for BE calls, we double the average total throughput of the cell. In addition, the increase in the mobility reserve $L_{R T}^{m}$ slightly decreases the total flow rate. This observation leads to the conclusion that a small amount of reserved sub channels for BE calls can reach a very high bit rate. In fact, by reserving some sub channels for BE calls, we also increase the probabilities of blocking and losing RT calls. Therefore, these amounts of resources released by blocked or lost calls allow BE calls to better utilize resources.

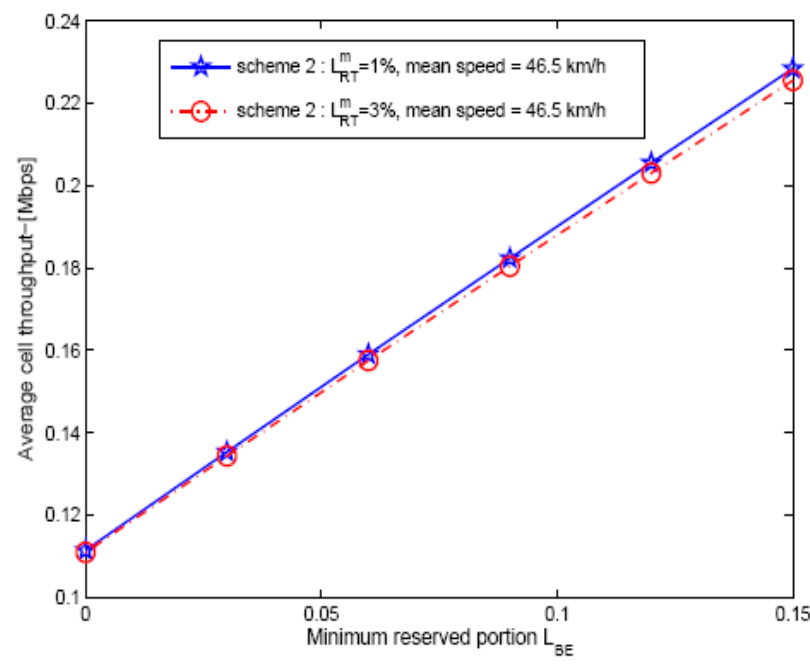

Fig. 7. Average total throughput

\section{CONCLUSION}

In this paper, we are interested in the quality of service offered by the IEEE802.16e system. We studied the continuous case of the IEEE802.16e system; we proposed an admission control algorithm or QoS management strategy in the IEEE802.16e system. This mechanism maintains the same quality of service for real-time calls and replaces best effort calls with fair share of resources, while reserving minimum bandwidth for best effort calls to ensure minimum QoS and minimum bandwidth real-time calls in intra-cell mobility. Moreover, we have implemented a more realistic model of mobility called RWP and that is often used in ad-hoc networks. We extend the RWP model in an IEEE802.16e cell subdivided into a finite number of regions. This model allowed us to calculate call migration rates between cell regions explicitly. On the basis of this proposition, we model our system using a CMTC continuous time Markov chain to find the stationary distribution of the system. Through the numerical results we were able to see the impact of this strategy on the performance of the system.

\section{REFERENCES}

[1] IEEE Standard for Local and Metropolitan Area Networks, Part 16: Air Interface for Broadband Wireless Access Systems IEEE Std 802.16 ${ }^{\mathrm{TM}_{-}}$ 2009.

[2] Universal Mobile Telecommunications System (UMTS); UE Radio Access capabilities (3GPP TS 25.306 version 11.8.0 Release 11), ETSI. January 2014.

[3] Technology of High Speed Packet Access (HSPA), White paper, EIKO SEIDEL, Nomor research Gmbh brecherspitzstr. 8- D 81541 Munchen, Germany, October 2006.

[4] IEEE Standard for local and Metropolitan Area Networks. IEEE $802.16 \mathrm{e} / \mathrm{d} 12$, air interface for fixed and mobile broadband wireless access systems.

[5] G. Karam,. Transmission techniques for digital terrestrial TV broadcasting. IEEE Communication Magazine, 33(2), 1995.

[6] IEEE Standard for local and Metropolitan Area Networks. IEEE 802.16-2004, air interface for fixed broadband wireless access systems.

[7] J. Li et al., Cell mobility based admission control for wireless networks with link adaptation. In proceeding of ICC,2007.

[8] F. Baskett, et al,. Open, closed and mixed networks of queues with different classes of customers. Journal ACM (JACM), 22 (2), pp: 248260, 1975.

[9] X. Chao, M. M, M. P, and B. Atkinson. Queuing networks : Customers, signals and product form solutions. The Journal of the Operational Research Society 52(5), pp. 600-601, 2001.

[10] S. Horrich, et al., On the impact of mobility and joint rrm policies on a cooperative WiMAX/hsdpa network. In proceeding of WCNC 2008.

[11] J. J. Won, et al., Perfomance analysis of random access protocol in OFDMA-CDMA. In proceeding of KICS Fall Conference, 2003.

[12] H. H. Seo, et al., A study of code partioning scheme of efficient random access in OFDMA-CDMA ranging subsystem. In proceeding of JCCI 2004, 262.

[13] T. Peyre, et al., QoS differentiation for initial and bandwidth request ranging in IEEE802.16. In proceeding of Personal, Indoor and Mobile Radio Communications Conference (PIMRC), September 2008.

[14] K. Wongthavarawat et al., Packet scheduling for QoS support in IEEE 802.16 broadband wireless access systems. International Journal of Communication Systems (16), pages 81-96, 2003.

[15] H. Wang, et al., Dynamic admission control and QoS for 802.16 wireless MAN. In proceeding of Wireless Telecommunications Symposium, 2005.

[16] C. Ball, et al., Performance analysis of temporary removal scheduling applied to mobile WiMAX scenarios in tight frequency reuse. In proceeding of International IEEE Symposium on Personal, Indoor and Mobile Radio Communications, 2005.

[17] E. Hyytiä et al,. Random waypoint mobility model in cellular networks. Wireless Network (13), pp: 177-188, 2007.

[18] N. Benameur, et al., Integrated admission control for streaming and elastic traffic. In proceeding of QOFIS, Coimbra, September 2001. 
[19] Q. Liu, et al., Queuing with adaptive modulation and coding over wireless links: Cross layer analysis and design. IEEE Transactions on wireless communications, 4(3), 2005.
[20] C. Tarhini et al., On capacity of OFDMA based IEEE802.16 WiMAX including adaptative modulation and coding and intercell intereference. In proceeding of LANMAN, Princeton, June 2007 